\title{
Contribution of genomic copy-number variations in prenatal oral clefts: a multicenter cohort study
}

\author{
Ye Cao, MD, PhD ${ }^{1,2}$, Zhihua Li, MD, PhD³, Jill A. Rosenfeld, MS4, \\ Amber N. Pursley, MS ${ }^{5,6}$, Ankita Patel, $\mathrm{PhD}^{5,6}$, Jin Huang, MD, PhD ${ }^{1}$, Huilin Wang, MS', \\ Min Chen, MD, PhD ${ }^{3}$, Xiaofang Sun, $\mathrm{PhD}^{3}$, Tak Yeung Leung, MD ${ }^{1,2}$, \\ Sau Wai Cheung, $\mathrm{PhD}^{5,6}$ and Kwong Wai Choy, PhD $1,2,7$
}

Purpose: We sought to investigate the utility of chromosomal microarray analysis (CMA) for prenatal diagnosis of oral clefts, as compared with traditional chromosome analysis, for improved prenatal genetic counseling and discovery of a potential correlation between genotype and oral cleft.

Methods: This retrospective analysis encompassed 270 prenatal oral cleft cases with documented detailed ultrasound findings and CMA results from four referral centers. Detection rates for pathogenic copy-number variants (CNVs) were calculated and compared with cases for which chromosome analysis was also performed.

Results: The overall detection rate was $14.8 \%$ (40/270) for pathogenic CNVs by CMA, $7.2 \%$ (9/125) for the nonsyndromic cases, and $21.4 \%$ $(31 / 145)$ for the syndromic cases. Of the nonsyndromic cases with ultrasound soft markers, $20 \%$ (5/25) were identified with pathogenic

Oral clefts are among the most common congenital birth malformations, with an incidence of $\sim 1$ in 700 live births. ${ }^{1}$ Although the exact causes of oral clefting are still inconclusive, both genetic and environmental factors are known to interfere with the highly regulated morphogenetic process that forms an intact oral cavity. ${ }^{2,3}$ Based on when the oral cavity fails to close during embryogenesis, the result is cleft lip, cleft lip and palate, or cleft palate. ${ }^{4}$ Oral clefts may be nonsyndromic, accounting for up to $70 \%$ of cases, or syndromic if there are concurrent congenital anomalies. ${ }^{1,5}$

Given the elevated infant mortality rates observed among oral cleft cases compared with the overall population, ${ }^{6}$ prenatal identification allows the expectant parents to make better informed health-care choices. Further, determination of the extent of clefting and whether it is truly an isolated lesion or part of a syndromic disease impacts the prognosis for the affected fetus and subsequent medical planning. Syndromic clefts are more frequently associated with chromosomal abnormalities, which are found by conventional chromosome
CNVs. CMA showed an improved detection rate of $15.3 \%(29 / 190)$ compared with $10.5 \%$ (20/190) for chromosome analysis.

Conclusion: This study not only highlights the improved detection of chromosomal defects by CMA in prenatal oral clefts but also deepens our understanding of oral clefts. The results suggest that CMA is highly recommended in prenatal invasive genetic testing not only for syndromic oral cleft cases but also for nonsyndromic cases with soft markers. Candidate genes including CRKL, AKAP8, SYDE1, BRD4 are worthy of further investigation regarding their role in human palatogenesis.

Genet Med advance online publication 25 February 2016

Key Words: aCGH; CMA; copy-number variation; oral cleft; prenatal diagnosis analysis in $50.7 \%$ compared with only $0.9 \%$ in cases of presumed isolated clefts. ${ }^{7}$ Not surprisingly, $\sim 86 \%$ of the detected chromosomal abnormalities were trisomy 13 and trisomy 18 . Recently, chromosomal microarray analysis (CMA) has been frequently applied to discover submicroscopic genomic imbalances prenatally, which are beyond the resolution of conventional chromosome analysis. Using such technology, Shaffer et al. ${ }^{8}$ found that $10.3 \%(14 / 136)$ of the prenatal cleft cases had clinically significant findings, 13 of which had genomic imbalances less than $10 \mathrm{Mb}$. The incidence of genomic imbalances was $9.1 \%(5 / 55)$ in the nonsyndromic group and $11.1 \%(9 / 81)$ in the syndromic group. ${ }^{8}$ Although these findings confirmed the significant association between chromosomal abnormalities and syndromic oral cleft cases, they also suggest that CMA could increase the detection of genomic imbalances for nonsyndromic cases. However, specific information regarding detection rates for each cleft category and its advantage over traditional chromosomal analysis is limited. Hence, our study aims to compare the detection rates of these two methodologies

The first two authors contributed equally to this work

${ }^{1}$ Department of Obstetrics and Gynecology, Prince of Wales Hospital, The Chinese University of Hong Kong, Hong Kong SAR, China; ${ }^{2}$ Shenzhen Research Institute, The Chinese University of Hong Kong, Shenzhen, China; ${ }^{3}$ Department of Obstetrics and Gynecology, Key Laboratory of Reproduction and Genetics of Guangdong Higher Education Institutes, The Third Affiliated Hospital of Guangzhou Medical University, Guangzhou, Guangdong, China; ${ }^{4}$ Signature Genomic Laboratories, PerkinElmer, Inc., Spokane, Washington, USA; ${ }^{5}$ Department of Molecular and Human Genetics, Baylor College of Medicine, Houston, Texas, USA; ${ }^{6}$ Medical Genetics Laboratories, Baylor College of Medicine, Houston, Texas, USA; ${ }^{7}$ Angsana Molecular \& Diagnostics Laboratory (HK) Ltd., Hong Kong SAR, China. Correspondence: Kwong Wai Choy (richardchoy@cuhk.edu.hk) 
Table 1 Summary of 270 oral cleft cases

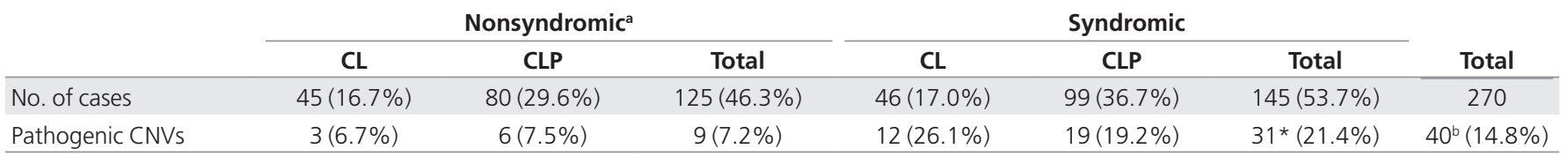

$\mathrm{CL}$, cleft lip only; CLP, cleft lip with palate; CNV, copy-number variant.

aral cleft plus soft marker cases were grouped into the nonsyndromic cleft category. An ultrasound soft marker was defined according to ref. 18. ${ }^{\circ}$ Two cases with incidental findings were excluded from the total. ${ }^{*} P=0.001$, Fisher's exact test. $P$ value assigned to the rate of pathogenic CNVs in the syndromic cases compared to the nonsyndromic cases.

and to provide a comprehensive summary of copy-number variants (CNVs) related to prenatal oral cleft cases. Such information will be useful for more informative genetic counseling and decision making regarding invasive prenatal diagnostic testing of fetuses presenting with oral clefts.

\section{MATERIALS AND METHODS}

This is a retrospective analysis of 270 prenatal oral cleft cases referred for CMA by four centers in Hong Kong SAR, mainland of China, and the United States between 2009 and 2012. Initially, a total of 292 prenatal oral cleft cases were referred for CMA. Twenty-one cases were excluded because either the specific type of oral cleft was not documented or the type of clefting reported (such as median cleft, atypical facial cleft, or Pierre Robin) results from a different pathogenesis. ${ }^{7}$ One additional case was excluded because the abnormal CMA result was suspected of being a cultural artifact due to extended cell culture time and was not confirmed by subsequent postnatal CMA. CMA was performed utilizing multiple platforms, ${ }^{9-12}$ including both oligonucleotide and bacterial artificial chromosome-based arrays (see details in the Supplementary Materials and Methods online). Quantitative polymerase chain reaction or fluorescence in situ hybridization was used to confirm the imbalances detected by CMA. Parental blood samples were collected when possible to assist with CNV interpretation and to exclude maternal cell contamination. CNVs were categorized as likely benign, pathogenic, or variant of unknown significance. Interpretation of $\mathrm{CNV}$ findings was performed in accordance with public and in-house databases, published cases, and clinical assessment by each center. G-banded chromosome analysis following standard protocols was performed or reported for 190 cases. Chromosome analysis was cancelled, not ordered, or results were not provided for the remainder of the cases. All the patients gave consent for analysis with local institutional ethic approval.

\section{RESULTS}

The cohort of 270 prenatal oral cleft cases was categorized into nonsyndromic and syndromic oral clefts and further subdivided into cleft lip only (CL) or cleft lip with palate (CLP), which also included cleft palate only, resulting in a total of four subgroups (Table 1): nonsyndromic CL (45/270, 16.7\%); nonsyndromic CLP (80/270, 29.6\%); syndromic CL (46/270, 17.0\%); and syndromic CLP (99/270, 36.7\%).

CNVs were reported for 66 of 270 (24.4\%) cases (Supplementary Table S1 online): 40 pathogenic CNVs (14.8\%),
14 variants of unknown significance (5.2\%), 10 likely benign (3.7\%), and 2 significant but incidental findings $(0.7 \%)$. The number of syndromic cases $(31 / 145,21.4 \%)$ identified with a pathogenic CNV was significantly higher $(P<0.05)$ than the number of nonsyndromic cases (9/125, 7.2\%; Table 1$)$. However, there was no statistically significant difference between the CL and CLP groups in either the nonsyndromic (6.7 vs. $7.5 \%)$ or the syndromic (26.1 vs. $19.2 \%$ ) groups.

Chromosome analysis results were available for 190 of the $270(70.4 \%)$ cases (Supplementary Table S2 online). Twenty cases $(10.5 \%)$ were detected with chromosomal abnormalities, including 5 cases with aneuploidy (25\%) and 15 cases with structural abnormalities (75\%). Two cases with suspected chromosomal abnormalities (46,XY,?5p and $47, \mathrm{XY},+\operatorname{mar}[14] / 46, \mathrm{XY}[1] \mathrm{dn})$ were reclassified as normal due to their normal CMA findings. Overall, the diagnostic yield of CMA $(29 / 190,15.3 \%, 95 \%$ confidence interval: $10.2-20.4 \%)$ was higher $(P=0.2205)$ than that of traditional chromosome analysis (20/190, 10.5\%, 95\% CI: 6.1-14.9\%). Although the detection rates for the syndromic and nonsyndromic groups were both improved by CMA, the syndromic group had a greater increase of $7.2 \%$ compared to the nonsyndromic group with an increase of $1.3 \%$. For both groups, CMA yielded a greater increase in detection of the CL cases as compared with the CLP cases. Of the remaining 80 cases without chromosome analysis results, $11(13.8 \%)$ had pathogenic CNVs, including 6 cases with CNVs $<10 \mathrm{Mb}$ that are not likely to be detectable microscopically.

To determine if specific concurrent ultrasound abnormalities were more likely to be associated with pathogenic CNVs, detection rates of pathogenic CNVs were calculated according to specific ultrasound findings. Of the 125 nonsyndromic cases, 25 cases had ultrasound soft marker findings (Table 2), 5 of which (20\%) had pathogenic CNVs. This rate is similar to the pathogenic CNV rate for syndromic clefts $(21.4 \%)$ and is also significantly higher $(P=0.02$, Fisher's exact test) than the rate of pathogenic CNVs among the nonsyndromic cases without a soft marker (4/100, 4.0\%). Among the syndromic cleft cases, CNS abnormalities (44.4\%), muscle and skeletal defects (34.7\%), and cardiac defects (32.6\%) were the most frequently associated structural anomalies (Supplementary Table S3 online), similar to the results other studies. ${ }^{13}$ The prevalence of pathogenic CNVs ranged from $11 \%$ to $42 \%$, with none of the ultrasound findings having a statistically significantly higher prevalence than the other concurrent abnormalities. 
Table 2 The prevalence of pathogenic CNVs in the nonsyndromic cases with or without ultrasound soft markers

\begin{tabular}{|c|c|c|c|}
\hline & $\mathrm{CL}$ & CLP & Totala \\
\hline No. of cases with soft markers & 10 & 15 & 25 \\
\hline Pathogenic CNV & $1(10 \%)$ & $4(26.7 \%)$ & $5(20 \%)$ \\
\hline No. of cases without soft markers & 35 & 65 & 100 \\
\hline Pathogenic CNV & $2(5.7 \%)$ & $2(3.1 \%)$ & $4(4.0 \%)$ \\
\hline
\end{tabular}

\section{DISSCUSION}

We evaluated the performance of CMA for detection of genomic alterations in a large cohort of prenatally detected oral clefts. Overall, 14.8\% (40/270) of the cases had a pathogenic CNV (Table 1). Our multicenter cohort demonstrated that CMA was useful for uncovering submicroscopic pathogenic CNVs, which were undetectable by traditional chromosomal analysis. CMA increased the detection rate of chromosomal imbalances by $4.8 \%$ in the 190 cases with both CMA and chromosome analysis results (Supplementary Table S2 online). The capability of CMA to provide a more precise diagnosis included detection of a single-gene disorder: a $0.16-\mathrm{Mb}$ Xq26.2 deletion in a male fetus (case 19) involving only one exon of the GPC3 gene and resulting in a diagnosis of Simpson-Golabi-Behmel syndrome (OMIM 312870). The precise diagnosis of prenatal cases has a profound impact on counseling and management and allows for better support for the family. Another striking example of the diagnostic capability of CMA is case 5 , who presented with an isolated CLP by ultrasound and a small $(1.6 \mathrm{Mb})$ deletion on $3 q$, revealing a diagnosis of $3 q 29$ deletion syndrome. Without access to the information provided by CMA, counseling would have included the possibility of a favorable outcome following the repair of the CLP. Rather, by having the knowledge that the fetus actually had a 3q29 deletion, more accurate counseling including the associated variable dysmorphic features, intellectual disability, and autism spectrum presentation ${ }^{14}$ was provided and allowed the parents to make more informed choices.

Further analysis of the cleft subgroups revealed several interesting observations about the prevalence of pathogenic CNVs. Unlike previous reports showing the lowest prevalence of chromosomal defects among the cleft lip-only cases, ${ }^{7}$ our data from the cases with both CMA and chromosome analysis performed (Supplementary Table S2 online) showed that pathogenic CNVs in the CL subgroups were unexpectedly higher than those of the CLP cases in both groups. This finding indicates that the enhanced detection provided by CMA is especially impactful for CL cases. Alternatively, some of the CLP cases may be mistaken for CL due to the limitations of prenatal ultrasound. Another interesting finding was that $20 \%$ of the nonsyndromic cases with soft markers were detected with pathogenic CNVs; this rate is comparable to that of syndromic cases (21.4\%). Interestingly, all three nonsyndromic cases with a single umbilical artery had pathogenic CNVs. This implies that soft markers may be indicative of those cases more likely to have a chromosomal abnormality. However, more samples should be recruited to confirm and evaluate which soft markers would have this diagnostic impact. Considering the major anomalies identified concurrently with the oral clefts, the prevalence of pathogenic CNVs was highest in cases with cardiac defects (23.4\%), but it was not statistically significantly higher than the other two most common concurrent abnormalities (Supplementary Table S3 online). Taken together, the data indicate that CMA is most likely to yield clinically useful information for syndromic cleft cases, regardless of specific concurrent anomalies, and for nonsyndromic cases with soft markers.

The higher-resolution analysis offered by CMA permits the opportunity to discover candidate genes related to oral clefting, further deepening our understanding of the development of the oral cavity. A genome-wide view of the CNVs detected in our study (trisomy 13 excluded) is presented in Supplementary Figure S1 online, which highlights candidate loci for further evaluation. We dissected the recurrent or rare pathogenic CNVs detected in our cohort and analyzed the relationship of disrupted candidate genes with oral clefting. A recurrent 17p13.3 duplication was detected in two syndromic CLP cases. Hypotheses about the etiology of oral clefting in 17p13.3 duplications include duplication of $Y W H A E^{15}$ and haploinsufficiency of $A B R$ at the duplication breakpoint in the $A B R$ region. ${ }^{16}$ Both $17 \mathrm{p} 13.3$ duplications in our cohort contained YWHAE, but only one interrupted $A B R$. Hence, we are inclined to think that overdosage of YWHAE is related to oral clefting with variable penetrance. An atypical 1.2$\mathrm{Mb}$ 22q11.2 deletion syndrome was detected in case 12 with syndromic CL, anal atresia, increased NT, and anotia. Unlike the $3-\mathrm{Mb}$ common or $1.5-\mathrm{Mb}$ nested DiGeorge syndrome deletions, TBX1 was not deleted in this case. However, this deletion disrupted CRKL, and in Crkl null mice, unfused shelves cause clefting, indicating its role in palatogenesis. ${ }^{17,18}$ We also found a rare duplication on 19p13.12 in case 31 that overlapped with the CNV of Decipher patient 249428 who presented with a high palate. Genes Syde1, Brd4, and Akap8 in this duplication are expressed in the branchial arches and frontonasal process at GD 10.5 of mouse embryos, which indicates their crucial role for palatogenesis. ${ }^{19}$ Although CMA assists in the identification of candidate genes, functional studies to validate the role of these genes in oral clefting are required.

In addition to the advantages of CMA, genomic information that was not intended or relevant was also uncovered. Two cases with incidental findings (cases 41 and 42) had deletions that are probably unrelated to the oral cleft but still clinically significant: a carrier for Cohen syndrome (OMIM 216550) associated with hypotonia, microcephaly, intellectual disability, and joint hypermobility due to multiple exon deletions in VPS13B, and a late-onset disorder called hereditary neuropathy with liability to pressure palsies (OMIM 162500) that is due to $17 \mathrm{p} 12$ deletion involving PMP22 gene. Taking scenarios like this into consideration, both pretest and posttest counseling should be emphasized when applying CMA prenatally.

In conclusion, the risk of pathogenic CNVs in prenatally detected oral clefts ranged from as low as $6.7 \%$ in the 
nonsyndromic CL subgroup to as high as $26.1 \%$ in the syndromic CL subgroup. The improvement in the detection rate of CMA over chromosome analysis seen in our study strongly supports the value of CMA as a first-tier prenatal invasive test for syndromic oral clefts and nonsyndromic oral clefts with soft markers. Finally, by scrutinizing the gene content of the recurrent and rare CNVs in our cohort, we found that genes such as CRKL, AKAP8, SYDE1, and BRD4, shown to have critical functions in palatogenesis in animal models but not previously prioritized in humans, may be new candidate oral clefting genes.

\section{SUPPLEMENTARY MATERIAL}

Supplementary material is linked to the online version of the paper at http://www.nature.com/gim

\section{ACKNOWLEDGMENTS}

This research was partly supported by the National Basic Research Program of China (2012CB944600), the National Natural Science Foundation of China (project 81370715), and the Guangdong Province Natural Science Foundation (project 2013040012653). The authors are grateful to all the colleagues and collaborators for their invaluable suggestions and help in this study.

\section{DISCLOSURE}

The Department of Molecular and Human Genetics at Baylor College of Medicine, The Department of Obstetrics \& Gynaecology at The Chinese University of Hong Kong and the Angsana Molecular \& Diagnostics Laboratory $(\mathrm{HK}) \mathrm{Ltd}$. derive revenue from clinical laboratory testing conducted at their laboratories.

\section{REFERENCES}

1. Dixon MJ, Marazita ML, Beaty TH, Murray JC. Cleft lip and palate: understanding genetic and environmental influences. Nat Rev Genet 2011;12:167-178.

2. Molina-Solana R, Yáñez-Vico RM, Iglesias-Linares A, Mendoza-Mendoza A, Solano-Reina E. Current concepts on the effect of environmental factors on cleft lip and palate. Int J Oral Maxillofac Surg 2013;42:177-184.

3. Simioni M, Araujo TK, Monlleo IL, Maurer-Morelli CV, Gil-da-Silva-Lopes VL. Investigation of genetic factors underlying typical orofacial clefts: mutational screening and copy number variation. J Hum Genet 2015;60:17-25.
4. Marazita ML. The evolution of human genetic studies of cleft lip and cleft palate. Annu Rev Genomics Hum Genet 2012;13:263-283.

5. Setó-Salvia N, Stanier P. Genetics of cleft lip and/or cleft palate: association with other common anomalies. Eur J Med Genet 2014;57:381-393.

6. Carlson L, Hatcher KW, Vander Burg R. Elevated infant mortality rates among oral cleft and isolated oral cleft cases: a meta-analysis of studies from 1943 to 2010. Cleft Palate Craniofac J 2013;50:2-12.

7. Maarse W, Rozendaal AM, Pajkrt E, Vermeij-Keers C, Mink van der Molen AB, van den Boogaard MJ. A systematic review of associated structural and chromosomal defects in oral clefts: when is prenatal genetic analysis indicated? J Med Genet 2012;49:490-498.

8. Shaffer $L G$, Rosenfeld JA, Dabell MP, et al. Detection rates of clinically significant genomic alterations by microarray analysis for specific anomalies detected by ultrasound. Prenat Diagn 2012;32:986-995.

9. Leung TY, Vogel I, Lau TK, et al. Identification of submicroscopic chromosomal aberrations in fetuses with increased nuchal translucency and apparently normal karyotype. Ultrasound Obstet Gynecol 2011;38:314-319.

10. Breman A, Pursley AN, Hixson P, et al. Prenatal chromosomal microarray analysis in a diagnostic laboratory; experience with $>1000$ cases and review of the literature. Prenat Diagn 2012;32:351-361.

11. Coppinger J, Alliman S, Lamb AN, Torchia BS, Bejjani BA, Shaffer LG. Wholegenome microarray analysis in prenatal specimens identifies clinically significant chromosome alterations without increase in results of unclear significance compared to targeted microarray. Prenat Diagn 2009;29:1156-1166.

12. Shaffer LG, Dabell MP, Fisher AJ, et al. Experience with microarray-based comparative genomic hybridization for prenatal diagnosis in over 5000 pregnancies. Prenat Diagn 2012;32:976-985.

13. Genisca AE, Frias JL, Broussard CS, et al. Orofacial clefts in the National Birth Defects Prevention Study, 1997-2004. Am J Med Genet A 2009;149A: $1149-1158$.

14. Città S, Buono S, Greco D, et al. 3q29 microdeletion syndrome: cognitive and behavioral phenotype in four patients. Am J Med Genet A 2013;161A: 3018-3022.

15. Tucker ME, Escobar LF. Cleft lip/palate associated with 17p13.3 duplication involving a single candidate gene (YWHAE). Clin Genet 2014;85: 600-601.

16. Curry CJ, Rosenfeld JA, Grant E, et al. The duplication 17p13.3 phenotype: analysis of 21 families delineates developmental, behavioral and brain abnormalities, and rare variant phenotypes. Am J Med Genet A 2013;161A:1833-1852.

17. Racedo SE, McDonald-McGinn DM, Chung JH, et al. Mouse and human CRKL is dosage sensitive for cardiac outflow tract formation. Am J Hum Genet 2015;96:235-244.

18. Moon AM, Guris DL, Seo JH, et al. Crkl deficiency disrupts Fgf8 signaling in a mouse model of 22q11 deletion syndromes. Dev Cell 2006;10:71-80.

19. Draaken M, Mughal SS, Pennimpede T, et al. Isolated bladder exstrophy associated with a de novo $0.9 \mathrm{Mb}$ microduplication on chromosome 19p13.12. Birth Defects Res A Clin Mol Teratol 2013;97:133-139. 